\title{
Eigenanalysis of some preconditioned Helmholtz problems $^{\star}$
}

\author{
Howard C. Elman, Dianne P. O'Leary \\ Computer Science Department and Institute for Advanced Computer Studies, University of \\ Maryland, College Park, MD 20742, USA; e-mail: \{elman,oleaery\}@cs.umd.edu
}

Received March 24, 1998 / Revised version received September 28, 1998

Dedicated to Olof Widlund, with gratitude, in celebration of his 60th birthday

Summary. In this work we calculate the eigenvalues obtained by preconditioning the discrete Helmholtz operator with Sommerfeld-like boundary conditions on a rectilinear domain, by a related operator with boundary conditions that permit the use of fast solvers. The main innovation is that the eigenvalues for two and three-dimensional domains can be calculated exactly by solving a set of one-dimensional eigenvalue problems. This permits analysis of quite large problems. For grids fine enough to resolve the solution for a given wave number, preconditioning using Neumann boundary conditions yields eigenvalues that are uniformly bounded, located in the first quadrant, and outside the unit circle. In contrast, Dirichlet boundary conditions yield eigenvalues that approach zero as the product of wave number with the mesh size is decreased. These eigenvalue properties yield the first insight into the behavior of iterative methods such as GMRES applied to these preconditioned problems.

Mathematics Subject Classification (1991): 65N22

\section{Introduction}

This paper is concerned with properties of the eigenvalues of matrices arising from discretization of the Helmholtz equation

$$
-\Delta u-k^{2} u=f \text {. }
$$

\footnotetext{
* This work was supported by the U.S. Office of Naval Research under grant N000149510338 and by the National Science Foundation under grants CCR 95-03126 and DMS-94231331

Correspondence to: H.C. Elman
} 
This problem is of fundamental use for the models of scattering of acoustic waves in fluids [8]. We will consider it on domains $\Omega \subset \mathbb{R}^{d}, d=1,2,3$, where $\Omega$ is either the unit interval, square, or cube, with Sommerfeld-like boundary conditions

$$
u_{n}-\mathrm{i} k u=0
$$

on $\partial \Omega$, where $u_{n}$ is the outward normal derivative.

Discretization of the problem (1)-(2) results in a linear system of equations

$$
\mathcal{A} u=f .
$$

where $\mathcal{A}$ is typically complex, non-Hermitian, indefinite, and in two or three dimensions, very large and sparse. In [1], we developed a set of preconditioning techniques for this problem in the three-dimensional case, and we demonstrated their effectiveness in a collection of numerical experiments on benchmark problems. Although the results in this work were encouraging, the indefiniteness of the matrices together with the large size of the threedimensional problems make it difficult to derive an analysis that explains the behavior of such techniques. The purpose of this paper is to introduce a methodology for computing the eigenvalues of various preconditioned operators associated with (3), and to use the computed eigenvalues to help explain the performance of the ideas presented in [1].

The approach under consideration for preconditioning derives from perturbation of the boundary conditions leading to $\mathcal{A}$. That is, we use a discrete Helmholtz operator (1) but replace the Sommerfeld-like conditions (2) on some subset of the boundary with either Dirichlet conditions (prescribed values of $u$ ) or Neumann conditions (prescribed values of $u_{n}$ ). Let the resulting preconditioning matrix be denoted $\mathcal{M}$. One great advantage of such preconditioners is that the preconditioning operation, i.e., the computation of the action of $\mathcal{M}^{-1}$, can be performed using a fast direct method in time proportional to the number of mesh points times a logarithmic factor [13]. Moreover, since the preconditioner differs from the matrix by a relatively low-rank operator (depending only on the number of grid points on the boundary), many of the eigenvalues of the preconditioned operator will be identically one, and therefore we expect good performance of Krylov minimization or projection methods such as GMRES [12] or QMR [4].

Thus, we seek an understanding of the nonunit eigenvalues of $\mathcal{M}^{-1} \mathcal{A}$. The methodology presented here is to examine the eigenvalues of onedimensional versions of these preconditioned problems, and then to bootstrap these results into expressions for eigenvalues for problems in two or three dimensions. This technique applies in the case where both the discretization matrix and the preconditioning matrix are tensor products of one-dimensional problems, so we consider finite difference discretizations. 
For problems in one dimension, the difference between the discrete Sommerfeld and perturbed operators is a matrix of rank two, and identification of the eigenvalues of the preconditioned problem entails the (trivial) computation of eigenvalues of a $2 \times 2$ matrix. We then show that in two and three dimensions, the eigenvalues of the preconditioned operators can be computed by solving a set of smaller eigenvalue problems derived by generalizing the approach used for one dimension. In particular, the computations entail the solution of eigenvalue problems for matrices of size at most $O(n)$, where $n$ is the number of grid points in one dimension. This enables the identification of the eigenvalues of the higher-dimensional problems, a task that would otherwise be computationally intractable for fine grids, especially in the three dimensional case. Using these computed values, we demonstrate a correlation between the performance of the various preconditioners as presented in [1] and the spectral properties of the preconditioned operators.

Manteuffel and Parter [9] and Joubert, Manteuffel, Parter, and Wong [7] have proven a very interesting series of results about problems similar to ours. In particular, if the preconditioner $\mathcal{M}$ and the given operator $\mathcal{A}$ are both discretizations of second-order elliptic operators, then the $L_{2}$ condition number of the preconditioned problem $\mathcal{A M}^{-1}$ is bounded independent of $h$ if and only if $\mathcal{M}$ and $\mathcal{A}$ have the same boundary conditions. Similarly, the $L_{2}$ condition number of $\mathcal{M}^{-1} \mathcal{A}$ is bounded independent of $h$ if and only if the adjoint problems $\mathcal{M}^{*}$ and $\mathcal{A}^{*}$ have the same boundary conditions. They also show results on the $H_{1}$ condition number. Unfortunately, these beautiful results do not directly yield insight into the behavior of iterative methods such as GMRES and QMR; see, for example, [10]. The first two statements say that the singular values of $\mathcal{A M} \mathcal{M}^{-1}$ and those of $\mathcal{M}^{-1} \mathcal{A}$ can behave quite differently. In contrast, the eigenvalues of $\mathcal{A} \mathcal{M}^{-1}$ are the same as those of $\mathcal{M}^{-1} \mathcal{A}$, and the convergence properties of GMRES and QMR are largely determined by these eigenvalues along with their eigenvectors. Hence the need for this study. For similar results applied to first order operators, see $[5,11]$.

A summary of the paper is as follows. In Sects. 2, 3 and 4, we locate the eigenvalues of the preconditioned problems in one, two and three dimensions, respectively. In Sect. 5, we show how the performance of the preconditioned GMRES algorithm for three-dimensional problems is correlated with eigenvalue distributions. The final section draws some conclusions.

\section{One-dimensional problems}

Let $\hat{A}_{S}$ be the $n \times n$ matrix derived from finite difference approximation to (1) and (2) using a mesh $x_{j}=j h, j=0,1, \ldots, n+1$, with $h=1 /(n+1)$. We use central differences for the second derivatives in (1), using discrete values 
$u_{j}$ to approximate $u\left(x_{j}\right)$. For the boundary conditions, we approximate the normal derivative by one-sided first-order accurate finite differences to obtain equations for $u_{0}$ and $u_{n+1}$, and we then use these equations to eliminate these values from the central differences for $-\Delta u=-u^{\prime \prime}$ at $x_{1}$ and $x_{n}$. We thus obtain the $n \times n$ matrix

$$
\hat{A}_{S}=\left[\begin{array}{cccccc}
\gamma_{S} & -1 & & & & \\
-1 & \beta & -1 & & & \\
& \cdot & \cdot & \cdot & & \\
& & \cdot & \cdot & \cdot & \\
& & & -1 & \beta & -1 \\
& & & & -1 & \gamma_{S}
\end{array}\right]
$$

where

$$
\begin{aligned}
\beta & =2-k^{2} h^{2} \\
\gamma_{S} & =2-k^{2} h^{2}-\frac{1+\mathrm{i} k h}{1+k^{2} h^{2}} .
\end{aligned}
$$

For this one-dimensional problem, we wish to determine the eigenvalues and eigenvectors of $M^{-1} \hat{A}_{S}$, where $M$ is a preconditioning matrix.

\subsection{Preconditioning by changing the boundary conditions}

Suppose we choose as our preconditioner the matrix corresponding to imposing different boundary conditions at the two endpoints. This yields the matrix

$$
\hat{M}=\left[\begin{array}{cccccc}
\gamma_{M} & -1 & & & & \\
-1 & \beta & -1 & & & \\
& \cdot & \cdot & \cdot & & \\
& & \cdot & \cdot & \cdot & \\
& & & -1 & \beta & -1 \\
& & & & -1 & \gamma_{M}
\end{array}\right],
$$

A similarity transformation that moves the first row and column of $\hat{A}_{S}$ and $M$ to the last will put the troublesome part of the matrices in the bottom right corner:

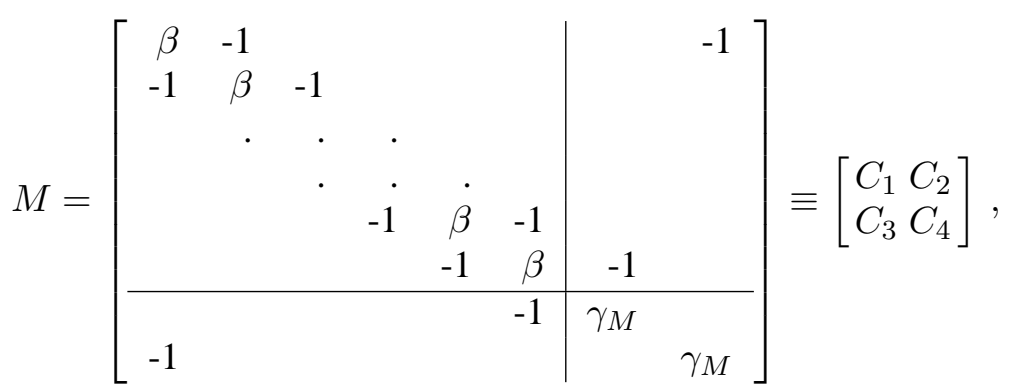




$$
A_{S}=\left[\begin{array}{rrrrrr|rr}
\beta & -1 & & & & & & -1 \\
-1 & \beta & -1 & & & & & \\
& \cdot & \cdot & \cdot & & & & \\
& & \cdot & \cdot & \cdot & & & \\
& & & -1 & \beta & -1 & & \\
& & & & -1 & \beta & -1 & \\
\hline-1 & & & & & -1 & \gamma_{S} & \\
& & & & & & \gamma_{S}
\end{array}\right] \equiv\left[\begin{array}{ll}
C_{1} & C_{2} \\
C_{3} & \bar{C}_{4}
\end{array}\right]
$$

Two lemmas provide the formulas we need to obtain the eigenvalues of the preconditioned problem.

Lemma 1. Given two matrices

$$
\mathcal{M}=\left[\begin{array}{ll}
C_{1} & C_{2} \\
C_{3} & C_{4}
\end{array}\right], \mathcal{A}=\left[\begin{array}{ll}
C_{1} & C_{2} \\
C_{3} & \bar{C}_{4}
\end{array}\right],
$$

where $\mathcal{M}$ and $C_{1}$ are nonsingular, then

$$
\mathcal{M}^{-1} \mathcal{A}=I+\left[\begin{array}{c}
-C_{1}^{-1} C_{2} S^{-1} \\
S^{-1}
\end{array}\right]\left[0 \bar{C}_{4}-C_{4}\right] \equiv I+U V^{*},
$$

where the Schur complement is defined by $S \equiv C_{4}-C_{3} C_{1}^{-1} C_{2}$.

Proof. The formula is verified by direct computation.

Lemma 2. If $U$ and $V$ are full-rank matrices of dimension $n \times p(p \leq$ $n$ ), then the matrix $U V^{*}$ has $n-p$ eigenvalues equal to zero, with right eigenspace equal to the orthogonal complement of the column space of $V$, and $p$ eigenvalues matching those of the $p \times p$ matrix $\Phi=V^{*} U$.

Proof. The matrix $U V^{*}$ is similar to the matrix $W U V^{*} W^{-1}$ for any nonsingular matrix $W$. Let

$$
W=\left[\begin{array}{l}
V^{*} \\
\bar{V}^{*}
\end{array}\right]
$$

where the columns of $\bar{V}$ form an orthonormal basis for the subspace orthogonal to the column space of $V$. Clearly, the $n-p$ columns of $\bar{V}$ span the null space of $\Phi$. If $V=Q R$, where the columns of $Q$ are orthonormal and the matrix $R$ is upper triangular, then

$$
W^{-1}=\left[Q R^{-*} \bar{V}\right]
$$

and

$$
W U V^{*} W^{-1}=\left[\begin{array}{cc}
\Phi & 0 \\
\bar{V}^{*} U & 0
\end{array}\right] .
$$

This matrix clearly has rank at most $p$, and the block triangular form tells us that the nonzero eigenvalues are those of $\Phi$. 
Corollary 1. Given $\mathcal{M}$ and $\mathcal{A}$ as in Lemma 1, the nonunit eigenvalues of $\mathcal{M}^{-1} \mathcal{A}$ are equal to $1+\mu$ where $\mu$ is an eigenvalue of $\Phi=V^{*} U=$ $\left(\bar{C}_{4}-C_{4}\right) S^{-1}$.

Applying the first lemma to our reordered matrices yields

$$
M^{-1} A_{S}=I+U V^{*},
$$

where

$$
U=\left[\begin{array}{c}
Z E S^{-1} \\
S^{-1}
\end{array}\right], V^{*}=\left[\begin{array}{ll}
0 & \delta I_{2 \times 2}
\end{array}\right] .
$$

Here $Z=C_{1}^{-1}$ is the inverse of the Toeplitz tridiagonal matrix with $\beta$ on the main diagonal and -1 above and below, $E=C_{2}$ is an $(n-2) \times 2$ matrix containing the last and first unit vectors,

$$
\delta=\left(\gamma_{S}-\gamma_{M}\right)
$$

and

$$
S=\gamma_{M} I_{2 \times 2}-E^{\mathrm{T}} Z E=\left[\begin{array}{cc}
\gamma_{M}-z_{n-2, n-2} & -z_{n-2,1} \\
-z_{1, n-2} & \gamma_{M}-z_{11}
\end{array}\right]
$$

Applying the second lemma, we see that $\Phi=\delta S^{-1}$, so its eigenvalues depend only on four elements of $Z: z_{11}=z_{n-2, n-2}$ and $z_{1, n-2}=z_{n-2,1}$. Computation of these eigenvalues yields $\phi_{11} \pm \phi_{12}$, so the eigenvalues of the preconditioned matrix are

$$
\lambda\left(A_{M}^{-1} A_{S}\right)=\left\{1+\phi_{11} \pm \phi_{12}, 1\right\} .
$$

This analysis requires that $\mathcal{M}$ and $C_{1}$ be nonsingular, which will not hold for certain values of $k$ and $h$ in the one-dimensional case. For the twoand three-dimensional problems, however, both of these matrices are always nonsingular due to the Sommerfeld boundary conditions on at least a portion of the boundary.

\subsection{Preconditioning using Neumann boundary conditions}

If we choose as our preconditioner the matrix $Q=\hat{A}_{N}$ corresponding to imposing Neumann boundary conditions at the two endpoints, approximating these conditions with a first order difference, then

$$
\gamma_{M}=1-k^{2} h^{2}
$$


Figure 1 displays the nonunit eigenvalues of $A_{N}^{-1} A_{S}$ for various values of $k$ and $n$, calculated using (4). ${ }^{1}$ As $n$ is increased, the eigenvalues come closer to each other, with the one with smaller real part staying relatively stationary near the value 1 and with the real part of the other one converging to 1 .

\subsection{Preconditioning using Dirichlet boundary conditions}

Suppose instead that we precondition by the matrix that corresponds to Dirichlet boundary conditions at both ends of the interval:

$$
M=\hat{A}_{D}=\left[\begin{array}{cccccc}
\beta & -1 & & & & \\
-1 & \beta & -1 & & & \\
& \cdot & \cdot & \cdot & & \\
& & \cdot & \cdot & \cdot & \\
& & & -1 & \beta & -1 \\
& & & & -1 & \beta
\end{array}\right] .
$$

In this case, $\gamma_{M}=\beta$. Using (4), we can determine the eigenvalues of the preconditioned matrix $\hat{A}_{D}^{-1} \hat{A}_{S}$. Figure 2 displays the two non-unit eigenvalues for various values of $k$ and $n$. As $n$ is doubled, the distance between these eigenvalues and the distance to the origin are both halved. As $k$ is increased, the eigenvalues move away from the origin and away from each other. The overall result is that an eigenvalue approaches zero as $k$ is decreased or $n$ is increased.

\section{Two-dimensional problems}

We now consider problem (1), (2) in two spatial coordinates. In this case, the coefficient matrix in (3) has the tensor product form

$$
\mathcal{A}=\hat{A}_{0} \otimes I+I \otimes \hat{A}_{S}
$$

and the preconditioners have the form

$$
\mathcal{M}=\hat{M}_{0} \otimes I+I \otimes \hat{A}_{S},
$$

where $\hat{A}_{0}=\hat{A}_{S}+k^{2} h^{2} I, \hat{M}_{0}=\hat{M}+k^{2} h^{2} I$, and $\hat{A}_{S}$ and $\hat{M}$ are as in the one-dimensional problem. Thus, the preconditioners are discretizations of the same differential operator but with different boundary conditions on two of the four edges of the unit square. This approach was first developed in

\footnotetext{
${ }^{1}$ If central differences are used for the boundary conditions, instead of one-sided differences, then the resulting matrices $A_{N}$ and $A_{S}$ are of dimension $(n+1) \times(n+1)$, with $\gamma_{N}=1-\frac{k^{2} h^{2}}{2}$ and $\gamma_{S}=\gamma_{N}-\mathrm{i} k h$. In this case, the matrix $\Phi$ is pure imaginary, so the real part of each eigenvalue is 1 , localizing all eigenvalues to the right half plane.
} 


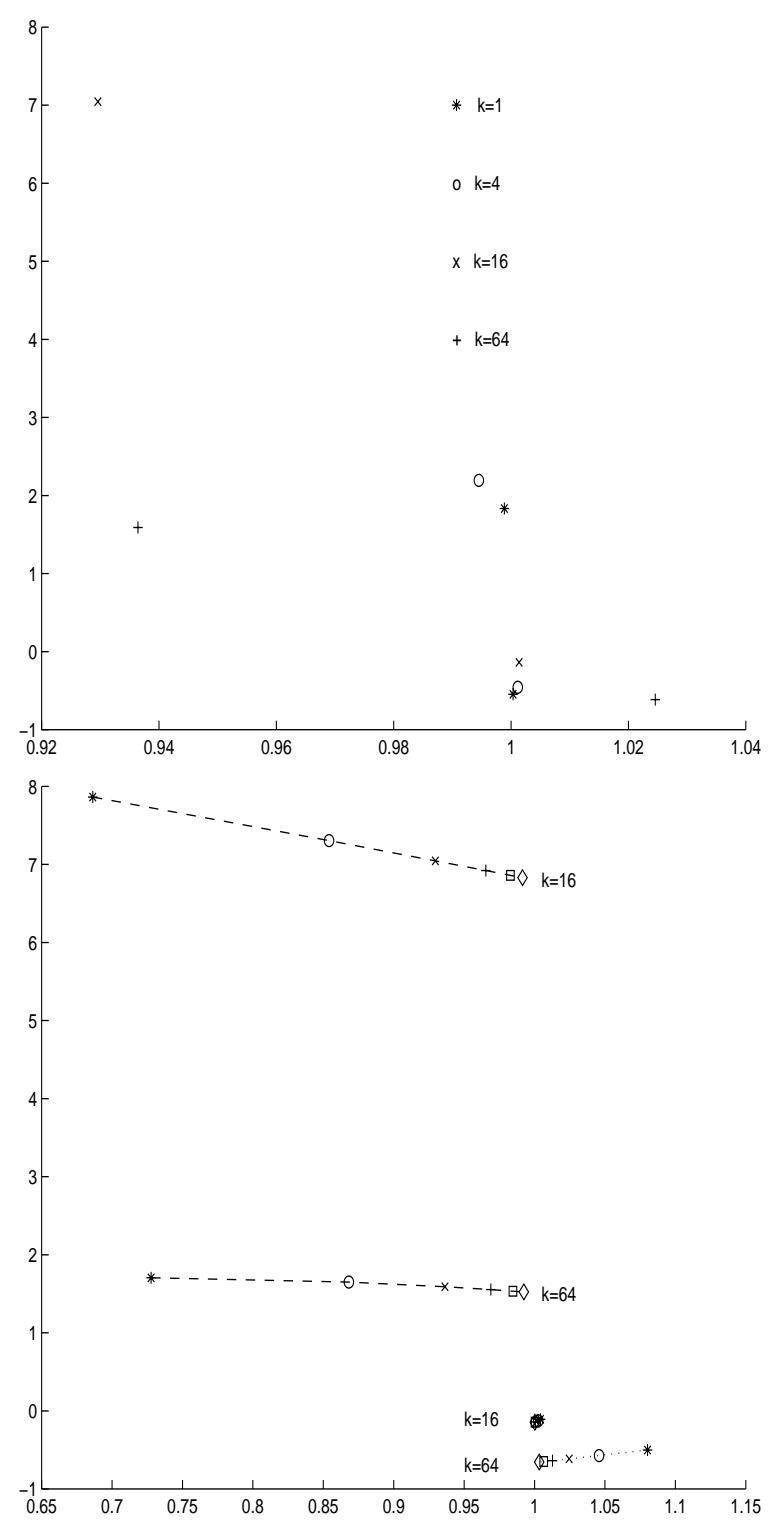

Fig. 1. The two nonunit eigenvalues of the 1-d Sommerfeld problem preconditioned by the 1-d Neumann. The top figure shows eigenvalues for values $k=1(*), k=4(\circ), k=16(\times)$ and $k=64(+)$ with $n=1600$. The bottom figure shows eigenvalues for values $n=400$ $(*), n=800(\circ), n=1600(\times), n=3200(+), n=6400(\square)$, and $n=12800(\diamond)$ with $k=16,64$. For each value of $k$, the maximal nonunit eigenvalues are connected by a dashed line and the minimal nonunit eigenvalues by a dotted line 


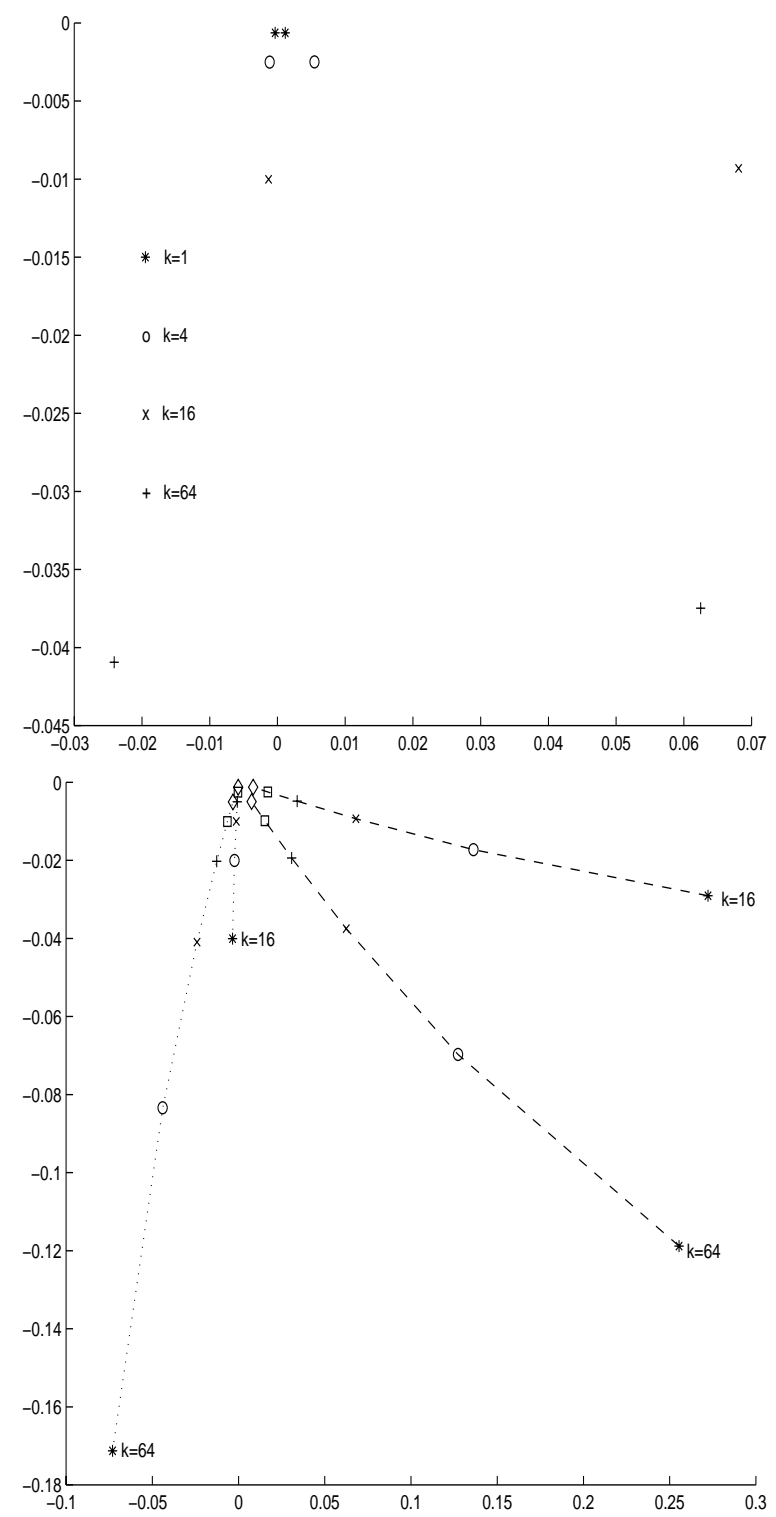

Fig. 2. The two nonunit eigenvalues of the 1-d Sommerfeld problem preconditioned by the 1-d Dirichlet. The top figure shows eigenvalues for values $k=1(*), k=4(\circ), k=16(\times)$ and $k=64(+)$ with $n=1600$. The bottom figure shows eigenvalues for values $n=400$ $(*), n=800(\circ), n=1600(\times), n=3200(+), n=6400(\square)$, and $n=12800(\diamond)$ with $k=16,64$. For each value of $k$, the maximal nonunit eigenvalues are connected by a dashed line and the minimal nonunit eigenvalues by a dotted line 
[2]. As above, we wish to determine the eigenvalues of the preconditioned matrix, which now takes the form

$$
\left(\hat{M}_{0} \otimes I+I \otimes \hat{A}_{S}\right)^{-1}\left(\hat{A}_{0} \otimes I+I \otimes \hat{A}_{S}\right) .
$$

Let $\hat{M}_{0}=F \Sigma F^{*}$, where $F$ is the matrix of eigenvectors and $\Sigma$ is diagonal. We simplify our matrix (5) somewhat through a similarity transformation using $F \otimes I$ to obtain

$$
\begin{aligned}
& \left(\Sigma \otimes I+I \otimes \hat{A}_{S}\right)^{-1}\left((\Sigma+R) \otimes I+I \otimes \hat{A}_{S}\right) \\
& =I+\left(\Sigma \otimes I+I \otimes \hat{A}_{S}\right)^{-1}(R \otimes I),
\end{aligned}
$$

where $R=F\left(\hat{A}_{S}-\hat{M}_{0}\right) F^{*}$ is a rank-2 matrix:

$$
\begin{aligned}
R & =\delta f_{1} f_{1}^{*}+\left(\gamma_{S}-q_{n n}\right) f_{n} f_{n}^{*} \\
& =\left(\gamma_{S}-q_{11}\right)\left(\left[f_{1} f_{n}\right]\left[\begin{array}{l}
f_{1}^{*} \\
f_{n}^{*}
\end{array}\right]\right) \\
& \equiv \delta G G^{*},
\end{aligned}
$$

and $f_{1}^{*}$ is the first row of the eigenvector matrix and $f_{n}^{*}$ is the last. Lemma 2 tells us how the eigenvalues of the matrix in (6) can be computed from those of a matrix of dimension $2 n \times 2 n$ :

$$
\begin{aligned}
& \left(\Sigma \otimes I+I \otimes A_{S}\right)^{-1}(R \otimes I) \\
& \quad=\delta\left(\Sigma \otimes I+I \otimes A_{S}\right)^{-1}(G \otimes I)\left(G^{*} \otimes I\right) \\
& \quad=\delta\left[\begin{array}{ccc}
\hat{A}_{S}+\sigma_{1} I & \\
\cdot & & \\
& \cdot & \\
& & \hat{A}_{S}+\sigma_{n} I
\end{array}\right]
\end{aligned}
$$

so we need the eigenvalues of the $2 n \times 2 n$ matrix

$$
\begin{aligned}
& C \equiv \delta\left(G^{*} \otimes I\right)\left[\begin{array}{lll}
\hat{A}_{S}+\sigma_{1} I & & \\
& \cdot & \\
& \cdot & \\
& & \hat{A}_{S}+\sigma_{n} I
\end{array}\right]^{-1}(G \otimes I) \\
& =\left[\begin{array}{cc}
\sum_{j=1}^{n} f_{j 1}^{2}\left(\hat{A}_{S}+\sigma_{j} I\right)^{-1} & \sum_{j=1}^{n} f_{j 1} f_{j n}\left(\hat{A}_{S}+\sigma_{j} I\right)^{-1} \\
\sum_{j=1}^{n} f_{j 1} f_{j n}\left(\hat{A}_{S}+\sigma_{j} I\right)^{-1} & \sum_{j=1}^{n} f_{j n}^{2}\left(\hat{A}_{S}+\sigma_{j} I\right)^{-1}
\end{array}\right] .
\end{aligned}
$$


Now the eigendecomposition of each of the matrices in the summation is of the form $\hat{A}_{S}+\sigma_{j} I=U\left(\Lambda+\sigma_{j} I\right) U^{-1}$, so each block of $C$ can be diagonalized by a similarity transformation involving the eigenvectors of $\hat{A}_{S}$ :

$$
\begin{aligned}
& {\left[\begin{array}{cc}
U^{-1} & 0 \\
0 & U^{-1}
\end{array}\right] C\left[\begin{array}{cc}
U & 0 \\
0 & U
\end{array}\right]} \\
& \quad=\left[\begin{array}{cc}
\sum_{j=1}^{n} f_{j 1}^{2}\left(\Lambda+\sigma_{j} I\right)^{-1} & \sum_{j=1}^{n} f_{j 1} f_{j n}\left(\Lambda+\sigma_{j} I\right)^{-1} \\
\sum_{j=1}^{n} f_{j 1} f_{j n}\left(\Lambda+\sigma_{j} I\right)^{-1} & \sum_{j=1}^{n} f_{j n}^{2}\left(\Lambda+\sigma_{j} I\right)^{-1}
\end{array}\right],
\end{aligned}
$$

and if we permute the rows and columns of this matrix by taking them in order $1, n+1,2, n+2, \ldots$, the problem breaks into $n$ eigenproblems of dimension $2 \times 2$ with entries

$$
\left[\begin{array}{ll}
s_{m} & t_{m} \\
t_{m} & s_{m}
\end{array}\right]
$$

with

$$
s_{m}=\sum_{j=1}^{n} \frac{f_{j 1}^{2}}{\lambda_{m}+\sigma_{j}}, \quad t_{m}=\sum_{j=1}^{n} \frac{f_{j 1} f_{j n}}{\lambda_{m}+\sigma_{j}} .
$$

Therefore, the eigenvalues are $s_{m} \pm t_{m}, m=1, \ldots, n$.

\subsection{Preconditioning using Neumann boundary conditions}

If we construct the preconditioning matrix using Neumann boundary conditions on two sides of the domain, then multiplication by the matrix $F$ corresponds to a discrete inverse cosine transform, and multiplication by $F^{*}$ corresponds to a discrete cosine transform. For $j=1, \ldots, n$, the eigenvalues of $\hat{M}_{0}$ are

$$
\sigma_{j}=4\left(\sin \frac{(j-1) \pi}{2 n}\right)^{2}
$$

and the eigenvector components are

$$
\begin{aligned}
& f_{j 1}=\frac{1}{\sqrt{n / 2}} \cos \frac{(j-1) \pi}{2 n} \\
& f_{j n}=\frac{1}{\sqrt{n / 2}} \cos \frac{(2 n-1)(j-1) \pi}{2 n}
\end{aligned}
$$

except that $f_{11}=f_{1 n}=1 / \sqrt{n}$. Figure 3 shows the location of the eigenvalues for the preconditioned problem for $n=50$ and for $n=500$. These computations were done using Matlab 4.2, since Matlab 5 does not correctly handle Kronecker products of sparse complex matrices. 

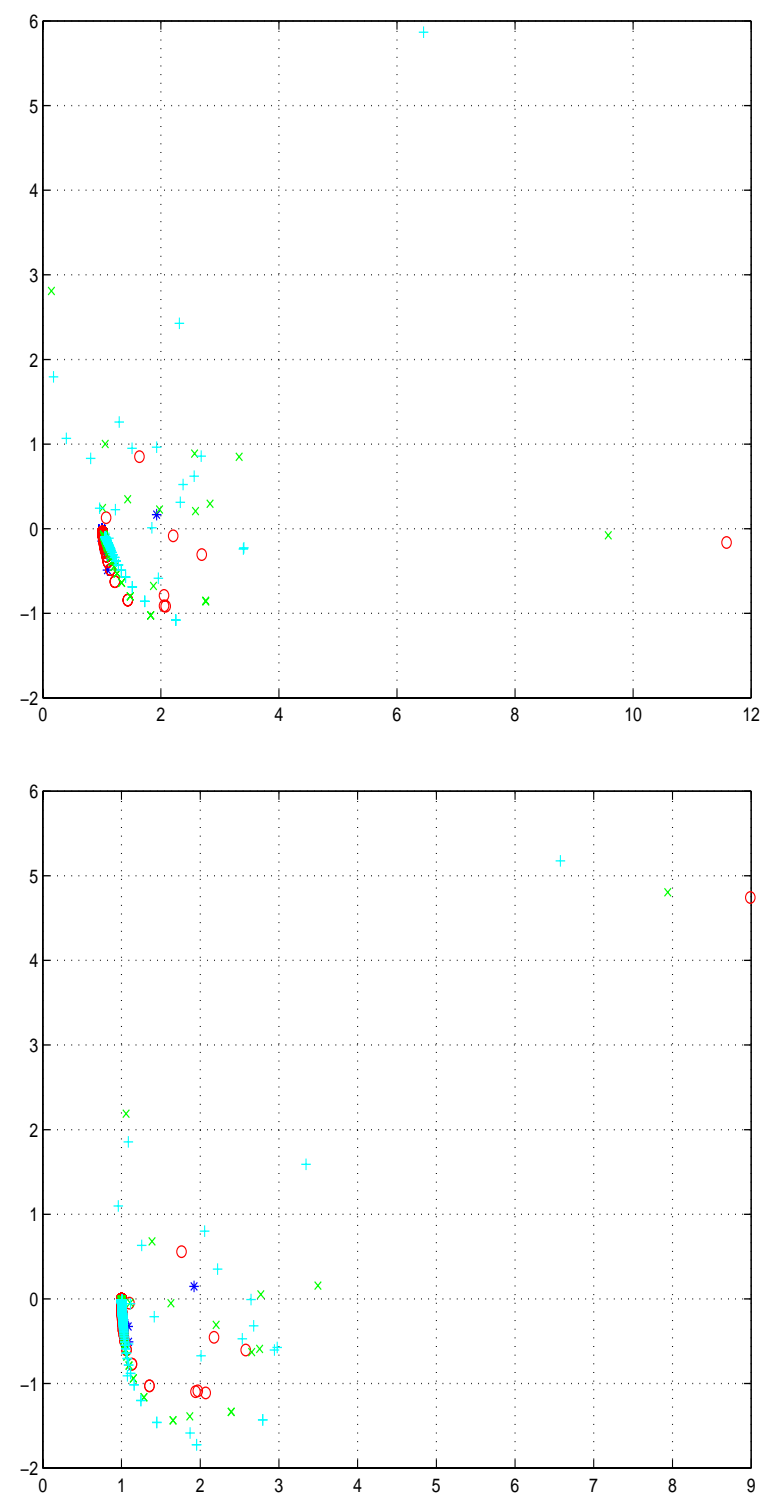

Fig. 3. Eigenvalues of the problem for a $50 \times 50$ grid ( 2500 unknowns, top) and $500 \times$ 500 grid (250, 000 unknowns, bottom) using a preconditioner with Neumann boundary conditions on two edges. Eigenvalues are displayed for $k=1\left({ }^{*}\right), k=10$ (o), $k=20(\times)$, and $k=30(+)$ 

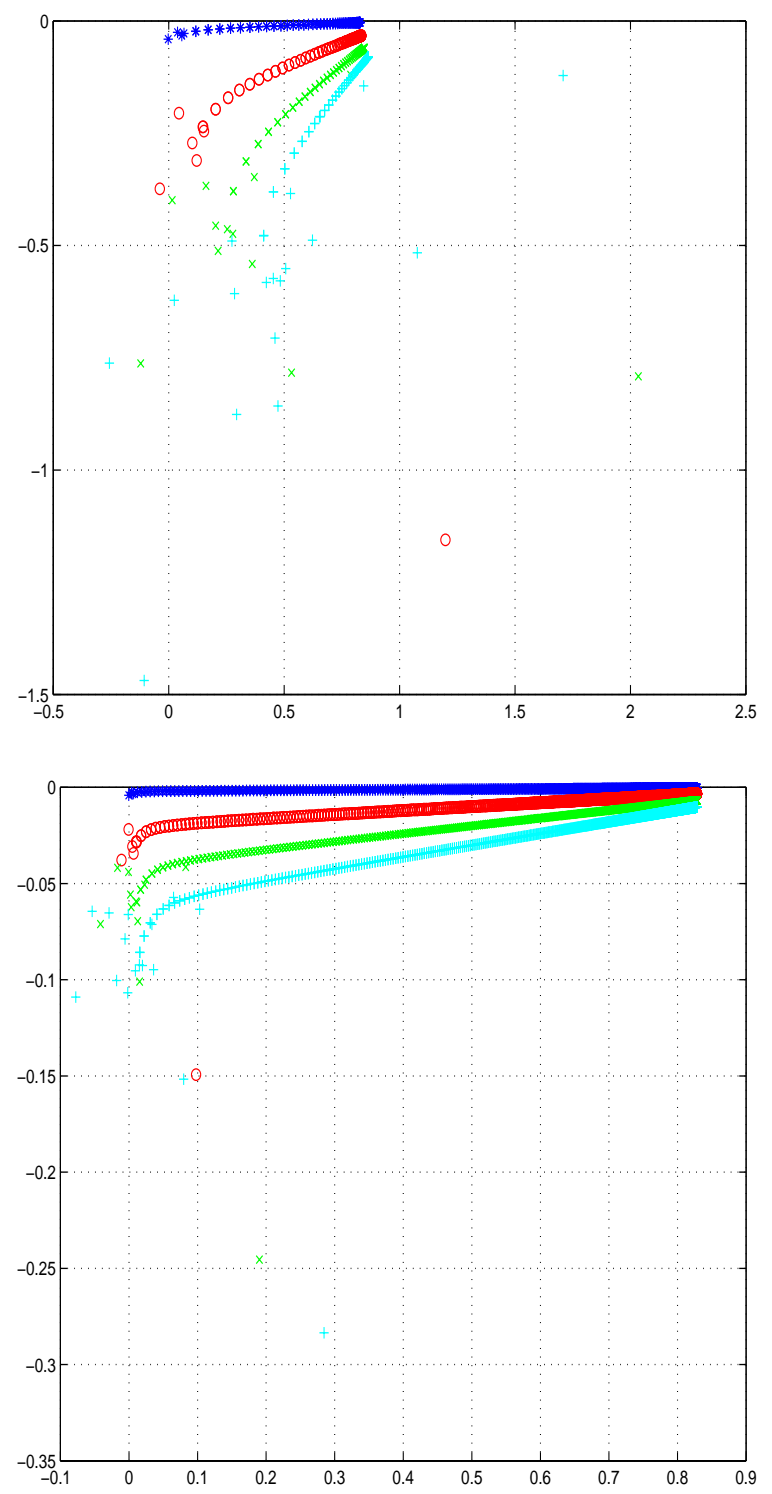

Fig. 4. Eigenvalues of the problem for a $50 \times 50$ grid ( 2500 unknowns, top) and a $500 \times 500$ grid (250, 000 unknowns, bottom) using a preconditioner with Dirichlet boundary conditions on two edges. Eigenvalues are displayed for $k=1(*), k=10(\mathrm{o}), k=20(\times)$, and $k=30$ (+) 
We note that all of the eigenvalues are in the right half plane, and that they remain outside the unit circle centered at the origin, except for $n=$ $50, k=30$, which has an eigenvalue 0.9939 from the origin. Note that this mesh size is generally considered to be too coarse for this value of $k$, since it has fewer than ten grid points per wavelength; the usual rule is to keep $k \leq 2 \pi /(10 h)[6]$.

\subsection{Preconditioning using Dirichlet boundary conditions}

If we construct the preconditioning matrix using Dirichlet boundary conditions on two sides of the domain, the situation is similar. Multiplication by the matrix $F$ corresponds to a discrete inverse sine transform, and multiplication by $F^{*}$ corresponds to a discrete sine transform. For $j=1, \ldots, n$, the eigenvalues of $\hat{M}_{0}$ are

$$
\sigma_{j}=4\left(\sin \frac{j \pi}{2(n+1)}\right)^{2},
$$

and the eigenvector components are

$$
\begin{aligned}
& f_{j 1}=\frac{1}{\sqrt{(n+1) / 2}} \sin \frac{j \pi}{n+1}, \\
& f_{j n}=\frac{1}{\sqrt{(n+1) / 2}} \sin \frac{j n \pi}{n+1} .
\end{aligned}
$$

Figure 4 shows the results for a $50 \times 50$ grid and for a $500 \times 500$ grid. Note that most eigenvalues lie on a smooth curve, with an accumulation point far from the origin and a few outliers. The curve moves away from the origin as $k$ increases and toward the origin as $n$ increases, and it has a slope of about $0.05 \frac{k}{n}$.

\section{Three-dimensional problems}

In three dimensions, we consider the discretized version of (1), (2) preconditioned by the discretized version of the same operator but with the Sommerfeld boundary conditions replaced by separable boundary conditions on four faces of the unit cube. The coefficient matrix is

$$
\hat{A}_{0} \otimes I \otimes I+I \otimes \hat{A}_{0} \otimes I+I \otimes I \otimes \hat{A}_{S},
$$

the preconditioner has the form

$$
\hat{M}_{0} \otimes I \otimes I+I \otimes \hat{M}_{0} \otimes I+I \otimes I \otimes \hat{A}_{S},
$$


and we wish to determine the eigenvalues of the preconditioned matrix

$$
\begin{aligned}
& \left(\hat{M}_{0} \otimes I \otimes I+I \otimes \hat{M}_{0} \otimes I+I \otimes I \otimes \hat{A}_{S}\right)^{-1} \\
& \quad \times\left(\hat{A}_{0} \otimes I \otimes I+I \otimes \hat{A}_{0} \otimes I+I \otimes I \otimes \hat{A}_{S}\right) .
\end{aligned}
$$

Again we can simplify our matrix through a similarity transformation using $F \otimes F \otimes I$ (recall that $\hat{M}_{0}=F \Sigma F^{*}$ is the eigendecomposition) to obtain

$$
\begin{aligned}
(\Sigma & \left.\otimes I \otimes I+I \otimes \Sigma \otimes I+I \otimes I \otimes \hat{A}_{S}\right)^{-1} \\
& \times\left((\Sigma+R) \otimes I \otimes I+I \otimes(\Sigma+R) \otimes I+I \otimes I \otimes \hat{A}_{S}\right) \\
= & I+\left(\Sigma \otimes I \otimes I+I \otimes \Sigma \otimes I+I \otimes I \otimes \hat{A}_{S}\right)^{-1} \\
& \times(R \otimes I+I \otimes R) \otimes I,
\end{aligned}
$$

where $R=F\left(\hat{A}_{S}-\hat{M}_{0}\right) F^{*}=\delta G G^{*}$ is a rank-2 matrix. Now $(R \otimes I+I \otimes R)$ is a matrix of rank $4(n-1)$ and can be expressed as $W Z^{*}$, where $W$ and $Z$ have $4(n-1)$ columns. (These columns span the row and column spaces and can be computed by the singular value decomposition or by taking $W$ to have the first $2(n-1)$ columns of $R \otimes I$ and columns $1,2, n+1, n+$ $2, \ldots,(n-3) n+1,(n-3) n+2,(n-1) n+1,(n-1) n+2$ from $I \otimes R$. $)$ We can now apply Lemma 2 to compute the eigenvalues of the preconditioned matrix from those of a matrix of dimension $4 n(n-1) \times 4 n(n-1)$ :

$$
\left(Z^{*} \otimes I\right)\left(\Sigma \otimes I \otimes I+I \otimes \Sigma \otimes I+I \otimes I \otimes \hat{A}_{S}\right)^{-1}(W \otimes I) .
$$

Just as in the 2-dimensional case, applying the similarity transformation that diagonalizes $\hat{A}_{S}$ breaks this eigenvalue problem into a set of smaller ones: in this case, $n$ problems of size $4(n-1)$. Using the second basis, these problems can be further decomposed, but we will not exploit this fact in the computations here.

\subsection{Preconditioning using Neumann boundary conditions}

Figure 5 shows the location of the eigenvalues for the preconditioned problem for $n=50$ and $n=80$. Again, the eigenvalues are all in the right half plane and nearly outside the unit circle.

\subsection{Preconditioning using Dirichlet boundary conditions}

Figure 6 shows the results for a $50 \times 50 \times 50$ grid and for a $80 \times 80 \times 80$ grid. All of the eigenvalues have negative imaginary part, and eigenvalues are closer to zero as $k / n$ decreases. 

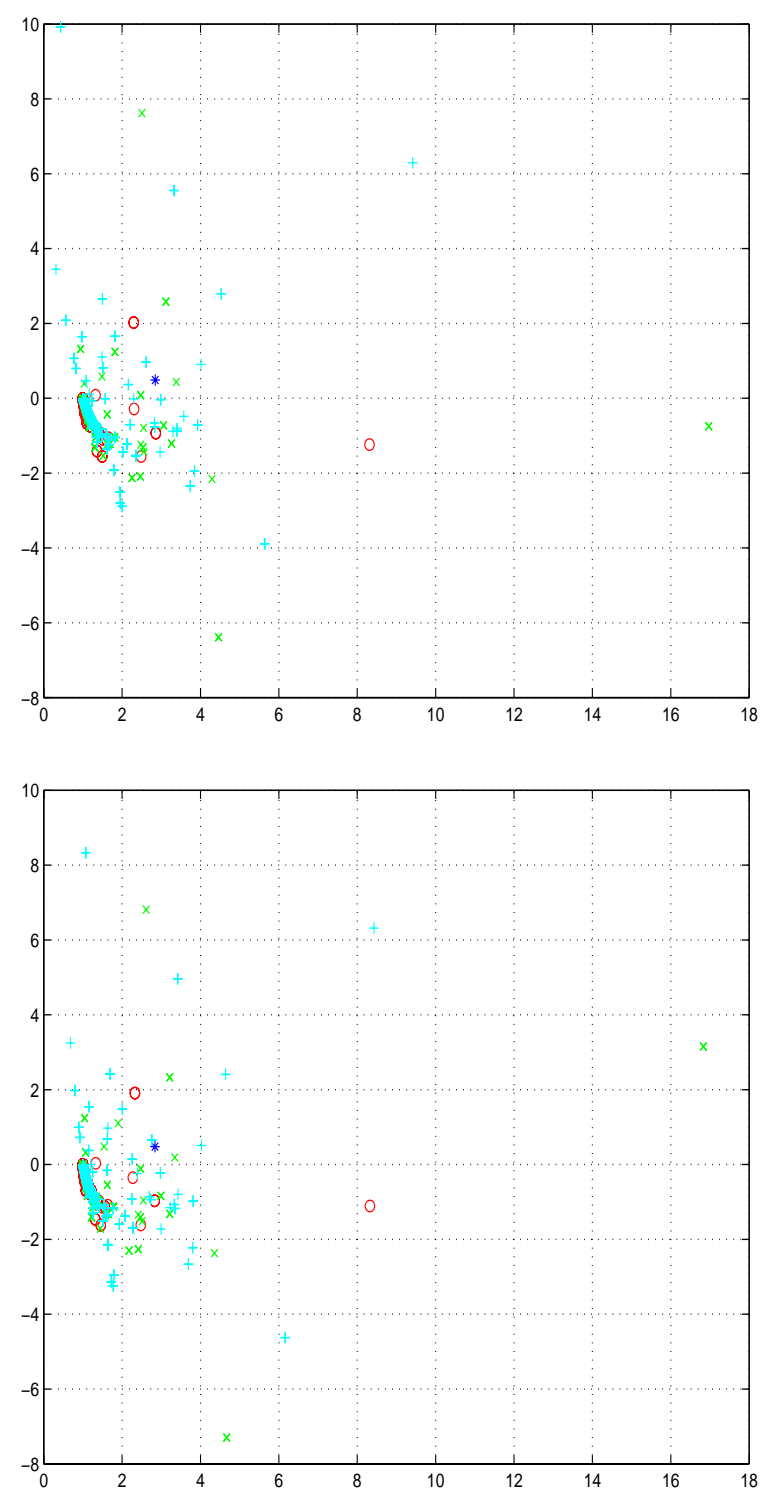

Fig. 5. Eigenvalues of the problem for a $50 \times 50 \times 50$ grid $(125,000$ unknowns, top) and $80 \times 80 \times 80$ grid $(512,000$ unknowns, bottom) using the Neumann preconditioner. Eigenvalues are displayed for $k=1\left(^{*}\right), k=5(\mathrm{o}), k=10(\times)$, and $k=15(+)$

\section{Correlation of spectra and GMRES iteration behavior}

Consider the preconditioned version of (3),

$$
\left[\mathcal{A M} \mathcal{M}^{-1}\right][\mathcal{M} u]=f
$$



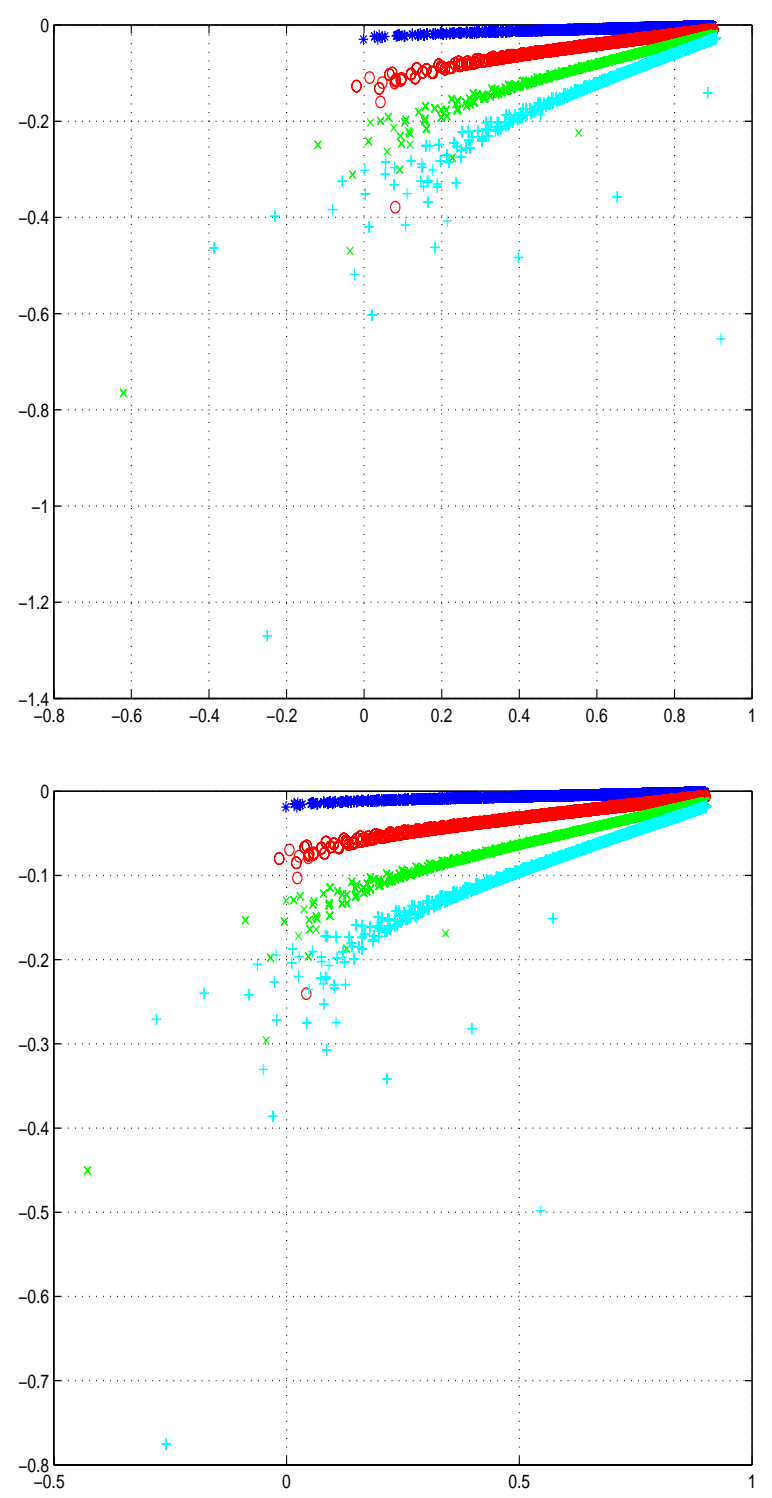

Fig. 6. Eigenvalues of the problem for a $50 \times 50 \times 50$ grid $(125,000$ unknowns, top) and $80 \times 80 \times 80$ grid $(512,000$ unknowns, bottom) using the Dirichlet preconditioner. Eigenvalues are displayed for $k=1\left({ }^{*}\right), k=5(\mathrm{o}), k=10(\times)$, and $k=15(+)$

Suppose the coefficient matrix is diagonalizable, i.e., $\mathcal{A M}^{-1}=V \Lambda V^{-1}$. It is well known that the GMRES algorithm produces a sequence of approximate solutions $u_{m}$ whose residuals $r_{m}=f-\mathcal{A} u_{m}$ satisfy

$$
\left\|r_{m}\right\|_{2}=\min \left\|V p_{m}(\Lambda) V^{-1} r_{0}\right\|_{2}
$$



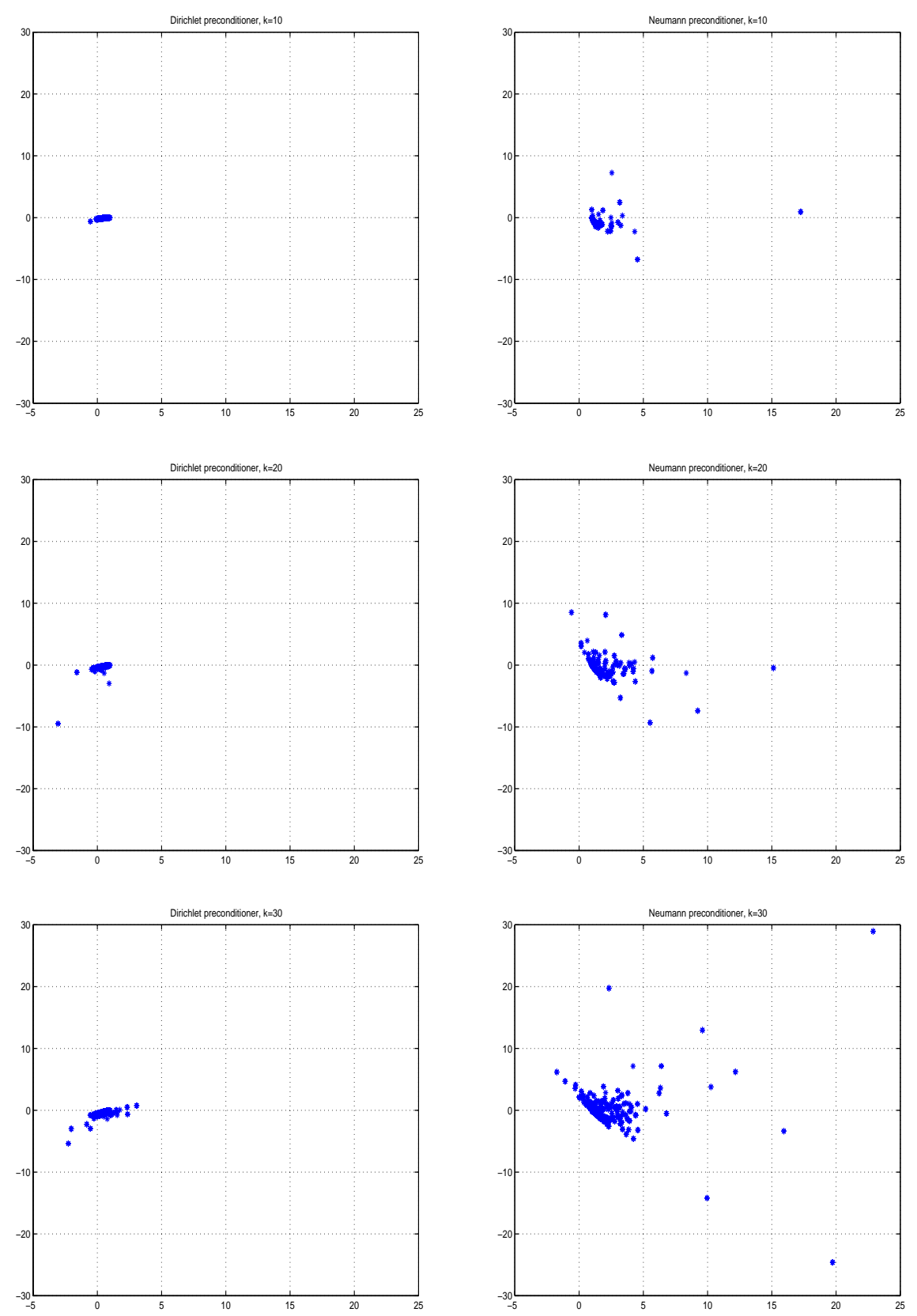

Fig. 7. Eigenvalues of preconditioned three-dimensional problems, for a $60 \times 60 \times 60$ grid 

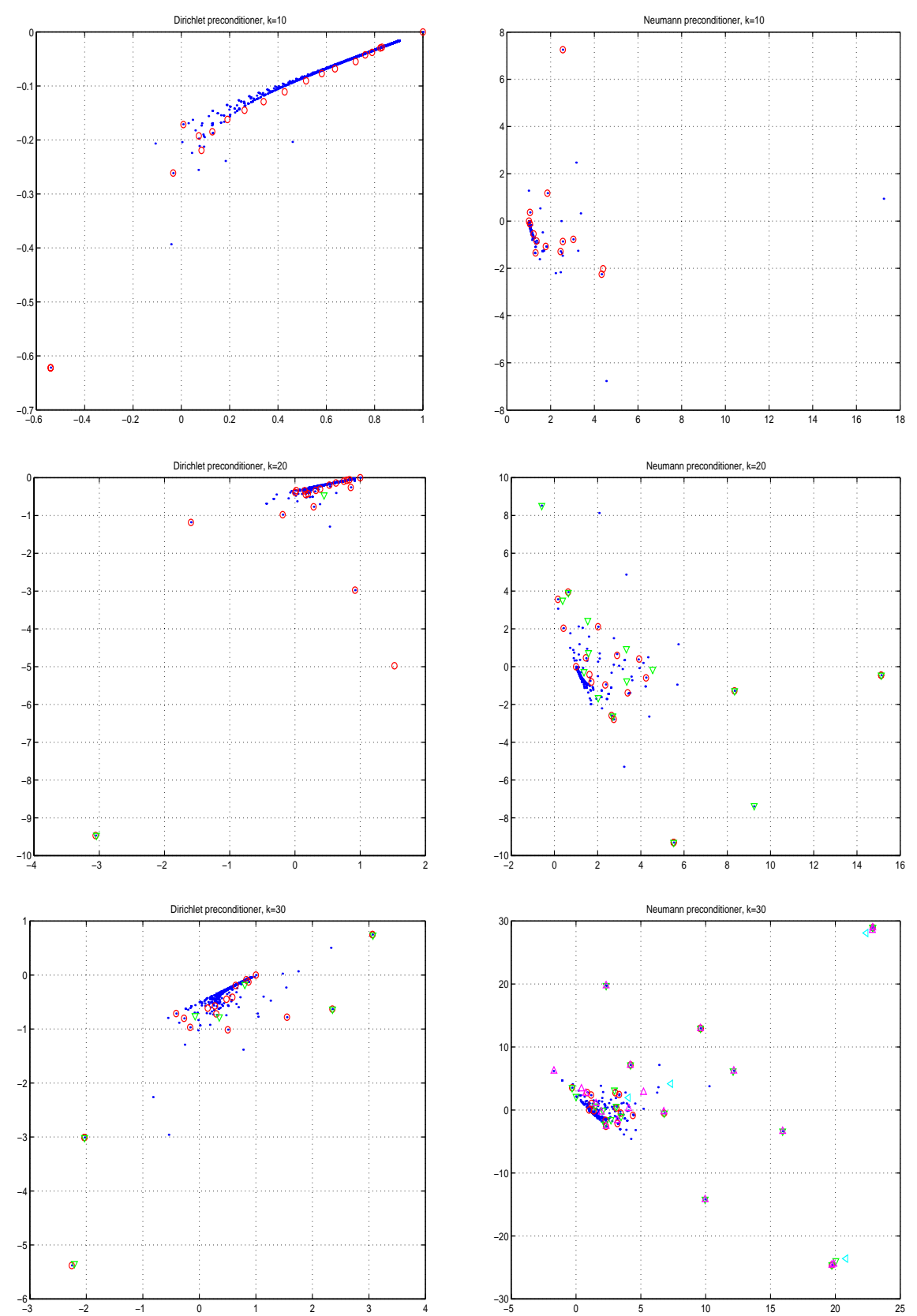

Fig. 8. Eigenvalues (.) and generalized Ritz values (०, $\nabla, \triangle, \triangleleft, \triangleright, \diamond)$ for $m=20$ of preconditioned three-dimensional problems, for a $60 \times 60 \times 60$ grid 

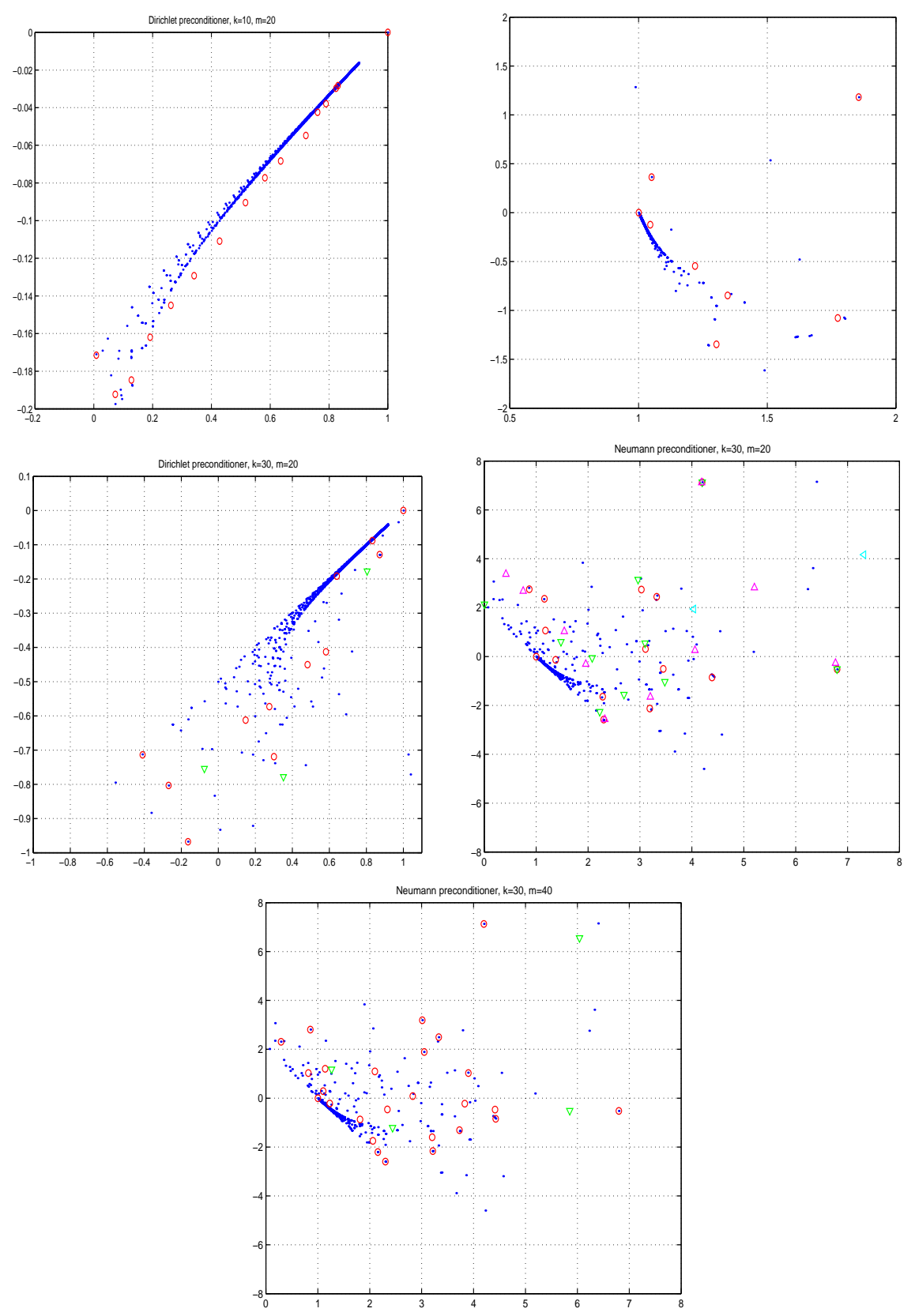

Fig. 9. Subsets of eigenvalues and generalized Ritz values of selected problems, for a $60 \times 60 \times 60$ grid 
Table 1. Iteration counts for GMRES(20) applied to the preconditioned discrete Helmholtz problem

\begin{tabular}{|c|c|c|c|c|c|c|c|c|c|}
\hline \multicolumn{5}{|c|}{ Dirichlet } & \multicolumn{5}{|c|}{ Neumann } \\
\hline & \multicolumn{4}{|c|}{$n$} & & \multicolumn{4}{|c|}{$n$} \\
\hline$k$ & 20 & 40 & 60 & 80 & $k$ & 20 & 40 & 60 & 80 \\
\hline 1 & 16 & 20 & 28 & 36 & 1 & 6 & 6 & 5 & 5 \\
\hline 5 & 13 & 16 & 20 & 23 & 5 & 9 & 9 & 9 & 9 \\
\hline 10 & 13 & 17 & 20 & 24 & 10 & 13 & 14 & 14 & 15 \\
\hline 20 & 14 & 16 & 22 & 24 & 20 & 35 & 36 & 35 & 34 \\
\hline 30 & 20 & 22 & 27 & 29 & 30 & 51 & 63 & 64 & 65 \\
\hline 40 & 19 & 41 & 36 & 34 & 40 & 89 & 82 & 97 & 97 \\
\hline 50 & 19 & 47 & 47 & 49 & 50 & 125 & 140 & 150 & 149 \\
\hline
\end{tabular}

where the minimum is over all polynomials of degree $m$ such that $p_{m}(0)=$ 1 . Thus, the values taken on by $p_{m}$ at the spectrum of $\mathcal{A M} \mathcal{M}^{-1}$ play a significant role. In this section, we explore the correlation between the spectra of the preconditioned operators $\mathcal{A M} \mathcal{M}^{-1}$ and the performance of $\operatorname{GMRES}(m)$, the restarted GMRES algorithm with restarts every $m$ steps.

We first present a table showing iteration counts of GMRES(20) (restarts every twenty steps), for a collection of three-dimensional preconditioned discrete Helmholtz problems (8) with various values of wave number $k$ and mesh size $n$, and both Dirichlet and Neumann preconditioners. The test problems were chosen so that the discrete solution has values

$$
u(x, y, z)=\mathrm{e}^{\mathrm{i} \frac{k}{2}|2 x-1|}(y(y-1))^{2}(z(z-1))^{2}
$$

at mesh points $(x, y, z)$. This function satisfies a weak formulation of equations (1)-(2) with $f=0$, and it exhibits wavelike behavior with wavelength $2 \pi / k$. The stopping criterion for the iterative solver was

$$
\frac{\left\|r_{j}\right\|_{2}}{\|f\|_{2}}<10^{-5}
$$

and the initial guess was $u_{0} \equiv 0$. Note from Table 1 that performance as the mesh size decreases clearly correlates with the trends observed for $h \rightarrow 0$ in Sect. 4. That is, the Neumann preconditioner, for which the eigenvalues appear to be bounded away from the origin, is insensitive to mesh size, whereas the Dirichlet preconditioner, for which there are eigenvalues approaching zero with $h$, declines somewhat in effectiveness as the mesh is refined. (Entries in the table above the jagged line correspond to problems with at least ten grid points per wave.)

Next, we focus on the three values $k=10,20,30$ and grid size $n=60$, for which the iteration counts are highlighted in Table 1. Here, the performance of the Dirichlet preconditioner degrades only slightly as $k$ decreases, 
whereas the Neumann preconditioner is better for $k=10$ but its performance deteriorates significantly for the larger values. Figure 7 shows the eigenvalues for these six problems, computed using the method of Sect. 4 . The six plots have the same scale. It is evident that the eigenvalues for the Dirichlet preconditioned problem are fairly insensitive to the wave number $k$, whereas the spectra for the Neumann preconditioner are spreading significantly as $k$ increases. These results are largely consistent with the iterative performance.

We explore these trends further in Figs. 8 and 9. Let $p_{m}$ now denote the iteration polynomial generated by $m$ steps of GMRES. It is shown in [3] that the roots of this polynomial are the eigenvalues of the matrix

$$
\left(\tilde{H}_{m}^{*}\right)^{-1}\left(H_{m}^{*} H_{m}\right)
$$

where $H_{m}$ is the rectangular upper Hessenberg matrix of dimension $(m+$ 1) $\times m$ generated by $m$ steps of the Arnoldi computation used for GMRES [12] , and $\tilde{H}_{m}$ is the square submatrix of $H_{m}$ obtained by removing the last row. We refer to these as the (generalized) Ritz values. The graphs in Fig. 8 show the Ritz values for each of the six problems. These occur in groups of twenty, corresponding to the sets of twenty steps of GMRES occurring between restarts; the groups of Ritz values are differentiated using different symbols as indicated in the caption, with $\circ$ denoting those in the first group, etc. The graphs in Fig. 9 show subsets of the data from Fig. 8 in more detail, for $k=10$ and $k=30$, and for one other test, using GMRES(40) for $k=30$ and the Neumann preconditioner.

In discussing these results, we will distinguish among three types of eigenvalues, loosely defined as follows:

- Group 1: those that seem to lie along a smooth curve.

- Group 2: those near group 1 but not in it.

- Group 3: the remaining eigenvalues.

For $k=10$ and $k=30$, the group 1 and 2 eigenvalues are shown in Fig. 9. The number of group 3 eigenvalues increases with $k$ but remains less than 35 .

For $k=10$ in Fig. 9, where both preconditioners result in very fast convergence, there are relatively few eigenvalues in group 2 . The group 1 eigenvalues are well approximated by using a small number of Ritz values. A polynomial of low degree fit to group 1 gives very small values on all of it, and placing just a few polynomial roots within group 2 is enough to produce small residual values. In contrast, for larger $k$ and especially for the Neumann preconditioner, there are significantly more eigenvalues in group 2, and they are more widely distributed in the plane. In this case, a polynomial of low degree fit to group 1 would have very large function 
values on the group 2 eigenvalues, and thus more of the GMRES polynomial roots must be concentrated in group 2 in order to reduce the residual significantly. Figure 8 indicates that even after multiple restarts, degree 20 is not large enough to approximate all of these group 2 eigenvalues well. We believe these observations account for the differences in performance of convergence of restarted GMRES in the examples under consideration.

Figures 10-12 refine these considerations by explicitly examining the absolute values of the iteration polynomials for the case $k=30$. Figures 10 and 11 show the results for the Neumann preconditioner and $m=20$ and 40 , respectively, and Fig. 12 shows the results for the Dirichlet preconditioner. The left sides of the figures show the values of the polynomial on the more than 14,000 eigenvalues in groups 1 and 2, and the right sides are for the group 3 eigenvalues. The horizontal axes are indices of the eigenvalues, which are sorted by increasing distance from the point $(1,0)$ in the complex plane. Thus, in the left sides of the figures, eigenvalues with larger indices tend to be those in group 2, or, for the Dirichlet preconditioner, those in group 1 furthest from the accumulation point. Multiple eigenvalues appear multiple times, except for the eigenvalue 1 , which has multiplicity $n(n-2)^{2}$ and is the first eigenvalue in the list.

We first note that the extreme (group 3) eigenvalues are not significantly affecting performance. For those that are not captured quickly by Ritz values, the iteration polynomials are very large; consequently, the corresponding eigenvectors cannot figure prominently in the initial residual.

In contrast, the polynomials have small values on the vast majority of the group 1 and 2 eigenvalues. Moreover, in each of the three cases, one $\operatorname{GMRES}(m)$ cycle nearly uniformly damps all of these eigenvalues, except some of those with larger indices. In the residual $r_{m}$ remaining after one cycle, the components of eigenvectors corresponding to the damped eigenvalues are much smaller, and subsequent iteration polynomials will be largely determined by the other eigenvalues. One consequence of this is that the iteration polynomials generated by cycles of $\operatorname{GMRES}(m)$ after the first are larger on the majority of eigenvalues than the first iteration polynomial.

The values of the iteration polynomials for $m=20$ are clearly much larger for the Neumann preconditioner (Fig. 10) than for the Dirichlet preconditioner (Fig. 12). We attribute this to the observations made above, that the magnitudes and number of eigenvalues in group 2 are much larger for the Neumann preconditioner. In contrast, degree 40 polynomials for the Neumann preconditioner (Fig. 11) are comparable to the degree 20 polynomials for the Dirichlet preconditioner (Fig. 12). This explains Fig. 13, which shows the history of residual norms and indicates that roughly the same number (1.8) of cycles is needed to solve this problem with these two preconditioners and restart parameters. 

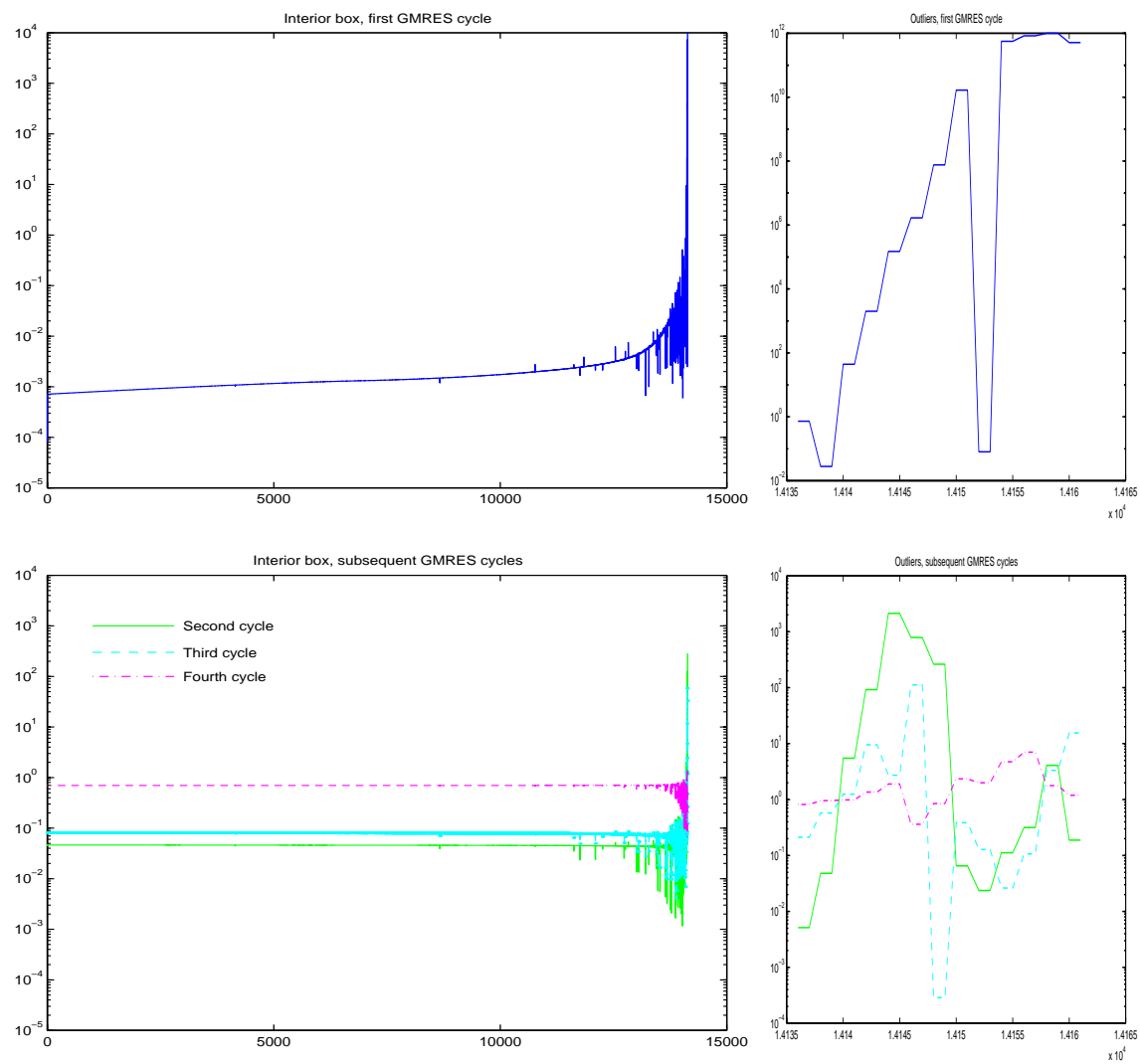

Fig. 10. Polynomial values $\left|p_{20}(\lambda)\right|$ for eigenvalues $\lambda$ of Neumann preconditioned operator, $k=30,60 \times 60 \times 60$ grid
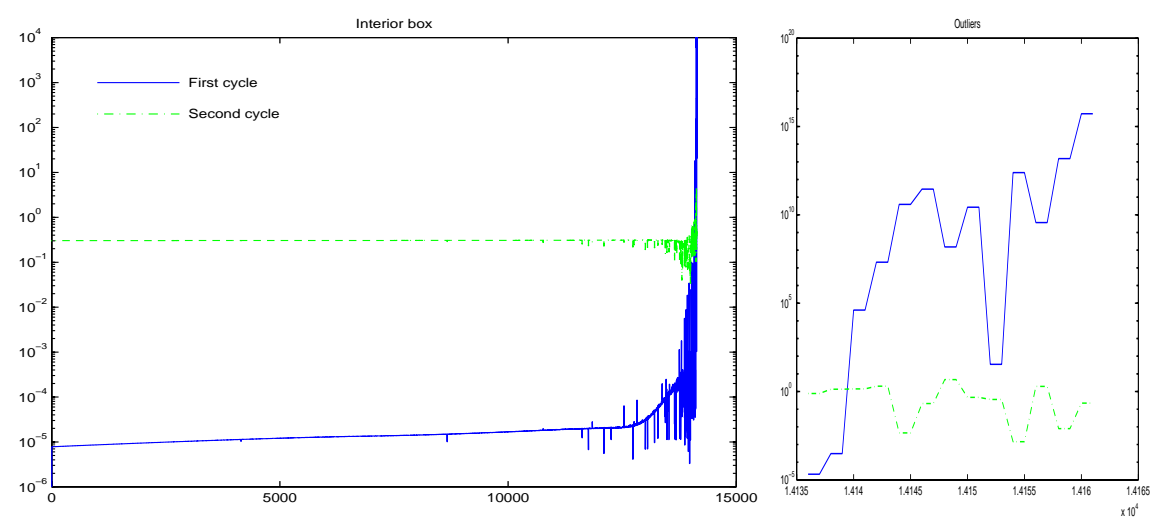

Fig. 11. Polynomial values $\left|p_{40}(\lambda)\right|$ for eigenvalues $\lambda$ of Neumann preconditioned operator, $k=30,60 \times 60 \times 60$ grid 

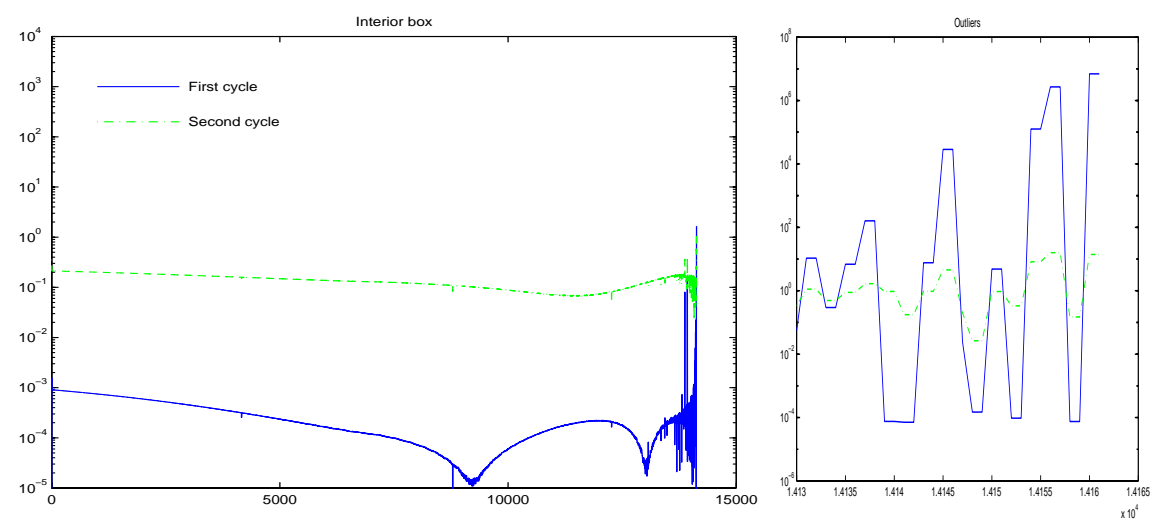

Fig. 12. Polynomial values $\left|p_{20}(\lambda)\right|$ for eigenvalues $\lambda$ of Dirichlet preconditioned operator, $k=30,60 \times 60 \times 60$ grid

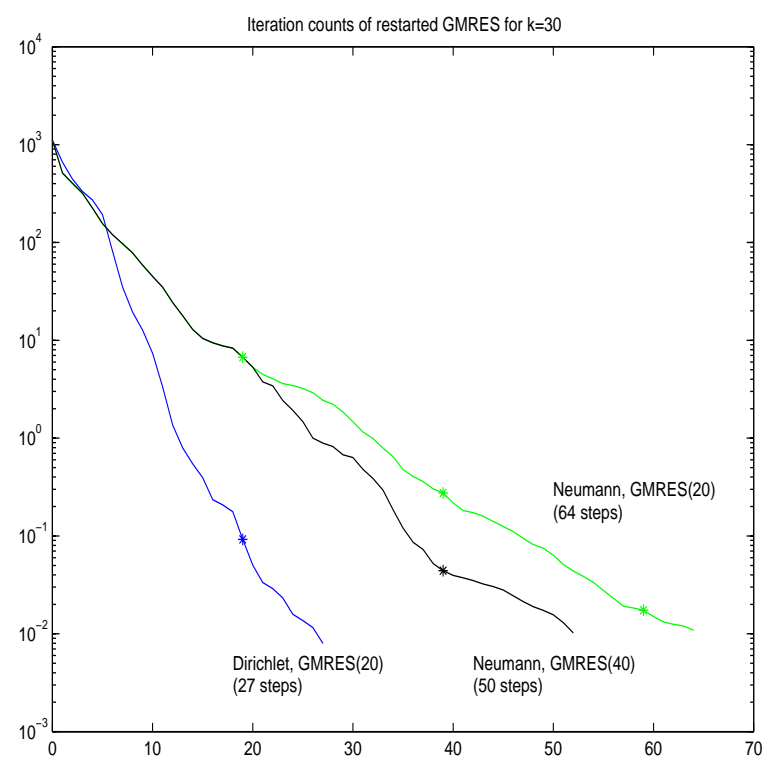

Fig. 13. Iteration counts of preconditioned restarted GMRES for $k=30,60 \times 60 \times 60$ grid

Finally, we contrast the results presented in this paper with some experiments performed previously and reported in [1]. There, we observed that when the Dirichlet preconditioner was used to solve problems with very smooth solutions, the sensitivity to mesh refinement (i.e., more iterations as $h$ decreases) was comparable to that displayed in Table 1 . In contrast, when this preconditioner was used to solve problems with a nonsmooth solution, the sensitivity to mesh refinement was considerably less pronounced. We have also examined the Ritz values for nonsmooth problems of this type, 
and found them to be less closely aligned with the eigenvalues of group 1, especially those far from the accumulation point. The results of Sects. 3-4 suggest that it is these eigenvalues that are tending to zero with $h$, and these observations indicate that the corresponding eigenvectors are in some sense smooth and figure less prominently in problems with nonsmooth solutions. For the benchmark problem (9) used here and for any fixed $k$, the solution looks smooth from the vantage point of all fine enough meshes as $h \rightarrow 0$, so it is not surprising that performance is like that for smooth problems. On meshes that are relatively coarse but still have several meshpoints per wave (i.e., those just above the jagged line of Table 1), the discrete solution (9) has a more oscillatory character. Performance in this case is indeed more like that for nonsmooth solutions; that is, the growth in iterations as we step down along the jagged line is slower than for very smooth solutions (cf. [1], Tables 2-3, left).

\section{Conclusions}

We have viewed some preconditioned Helmholtz problems from a capacitance matrix viewpoint, popularized by Olof Widlund, which exploits the structure of matrices of the form identity plus a low rank matrix. These discrete problems are quite difficult to analyze, because the usual tools related to positive definiteness or M-matrix properties are lacking. Using the capacitance matrix viewpoint, we have been able to explicitly calculate the eigenvalues of 1,2 , and 3 dimensional problems by solving a set of 1 dimensional eigenvalue problems. These calculations have revealed that the Dirichlet preconditioned matrix has eigenvalues that approach zero as the mesh size is decreased, while those of the Neumann preconditioned matrix are more dispersed but bounded away from zero. We have been able to use this insight to explain the behavior of GMRES on these problems.

Future work will extend these preconditioners to the case of non-constant wave number $k$.

Acknowledgements. We are grateful to Oliver Ernst for helpful discussions.

\section{References}

1. Elman, H.C., O'Leary, D.P. (1998): Efficient iterative solution of the three-dimensional Helmholtz equation. J. Comput. Physics 142, 163-181

2. Ernst, O., Golub, G.H. (1994): A domain decomposition approach to solving the Helmholtz equation with a radiation boundary condition. In: Quarteroni, A., Periaux, J., Kuznetsov, Y., Widlund, O. (eds) Domain Decomposition in Science and Engineering, pages 177-192. American Mathematical Society, Providence 
3. Freund, R.W. (1992): Quasi-kernel polynomials and their use in non-Hermitian matrix iterations. J. Comput. Appl. Math. 43, 135-158

4. Freund, R.W., Nachtigal, N.M. (1991): QMR: a quasi-minimal residual method for non-Hermitian linear systems. Numer. Math. 60, 315-339

5. Hemmingsson, L., Otto, K. (1996): Analysis of semi-Toeplitz preconditioners for firstorder PDEs. SIAM J. Sci. Comput. 17, 47-64

6. Ihlenburg, F., Babuška, I. (1995): Finite element solution of the Helmholtz equation with high wave number. Part I: The h-version of the FEM. Computers Math. Applic. 30(9), 9-37

7. Joubert, W., Manteuffel, T.A., Parter, S.V., Wong, S.-P. (1992): Preconditioning secondorder elliptic operators: Experiment and theory. SIAM J. Sci. Stat. Comput. 13, 259-288

8. Junger, M.C., Feit, D. (1986): Sound, Structures and their Interaction. MIT Press, Cambridge, MA, 2nd edition

9. Manteuffel, T.A., Parter, S.V. (1990): Preconditioning and boundary conditions. SIAM J. Numer. Anal. 27, 656-694

10. Nachtigal, N.M., Reddy, S.C., Trefethen, L.N. (1992): How fast are nonsymmetric matrix iterations. SIAM J. Matr. Anal. Appl. 13, 778-795

11. Otto, K. (1996): Analysis of preconditioners for hyperbolic partial differential equations. SIAM J. Numer. Anal. 33, 2131-2165

12. Saad, Y., Schultz, M.H. (1986): GMRES: A generalized minimal residual algorithm for solving nonsymmetric linear systems. SIAM J. Sci. Stat. Comput. 7, 856-869

13. Van Loan, C. (1992): Computational Frameworks for the Fast Fourier Transform. SIAM, Philadelphia 